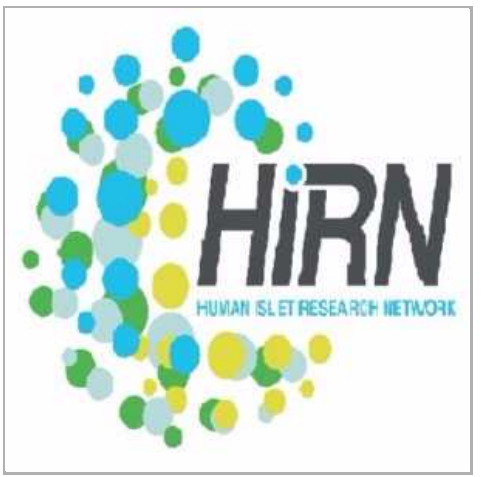

SEP 24, 2021

open ठAccess

DOI:

dx.doi.org/10.17504/protocol s.io. bvrfn53n

Protocol Citation: Mohsen Khosravi-Maharlooei, Markus Holzl, Austin Chen, Megan Sykes 2021. A General Guide To Generate Different Humanized Mouse Models. protocols.io

https://dx.doi.org/10.17504/p rotocols. io. bvrfn53n

License: This is an open access protocol distributed under the terms of the Creative Commons Attribution License, which permits unrestricted use, distribution, and reproduction in any medium, provided the original author and source are credited

Protocol status: Working We use this protocol and it's working

Created: Jun 11, 2021

Last Modified: Sep 24, 2021

PROTOCOL integer ID: 50695

1 NOD-scid common gamma chain knockout (NOD.Cg-Prkdcscid II2rgtm1Wjl/SzJ) (NSG) and NSG$\left(K^{b} D^{b}\right)^{\text {null }}(I A)^{\text {null }}(N S G$ MHC KO) mice are obtained from the Jackson Laboratory or bred in-house

\section{(3) A General Guide To Generate Different Humanized Mouse Models}

\author{
Mohsen Khosravi-Maharlooei ${ }^{1}$, Markus Holzl ${ }^{1}$, Austin Chen ${ }^{1}$, \\ Megan Sykes ${ }^{1}$ \\ ${ }^{1}$ Columbia Center for Translational Immunology, Columbia University, New \\ York
}

\section{Human Islet Research Network}

\section{Lili Liang}

\section{ABSTRACT}

This protocol details how to create humanized mouse models from NSG mice. Four different variants of humanized mice can be generated based on whether or not the native thymus is retained or if a human thymus piece is transplanted. Which type of humanized mouse is desired depends on the goals for the experiment. See our protocols on NSG mouse thymectomy, human CD34+ cell isolation, and human fetal thymus preparation for more details on some of the steps in this protocol ("Thymectomy procedure to remove native thymus of NSG mice", "Human CD34+ cell isolation from fetal liver, and fetal thymus preparation", "CD34+ isolation from human bone marrow").

Note

\section{Corresponding Authors}

\section{Mohsen Khosravi-Maharlooei}

Email:mkm2182@cumc.columbia.edu

\section{Austin Chen}

Email: ac4274@cumc.columbia.edu

Tel: 425-283-6900 
and housed in a specific pathogen-free microisolator environment. Adult mice are used at 6-10 wk of age.

2 Discarded human fetal thymus and liver tissues of gestational age 17-20 wk are obtained without identifiers from Advanced Bioscience Resources (ABR, Alameda, CA). Informed consent from women choosing to donate fetal remains for research are obtained by ABR.

3 Fetal thymus fragments are cryopreserved in 10\% dimethyl sulfoxide (DMSO) and $90 \%$ human AB serum (Atlanta Biologicals).

4 Fetal livers (FL) are cut into small pieces and incubated in $\triangle 5 \mathrm{~mL}$ of $[\mathrm{M}] 0.1 \mathrm{mg} / \mathrm{mL}$ liberase (Liberase ${ }^{T M}$ Research Grade, 05401119001, Roche) for 20 minutes in a $8^{\circ} 37^{\circ} \mathrm{C} \quad \mathrm{CO}_{2}$ incubator. Every 5-7 minutes, the samples are vortexed and pipetted up and down to facilitate the digestion process. Digested cells are washed and $\mathrm{CD}_{3}{ }^{+}$cells (referred to as $\mathrm{HSCs}$ ) are positively sorted with magnetic cell sorting (MACS) (Miltenyi Biotec). From each FL, we harvest 10-30 million CD34 ${ }^{+}$cells with a purity of $70-90 \%$. CD $34^{+}$HSCs are also cryopreserved in $10 \%$ DMSO and $90 \%$ human $A B$ serum.

5 Four types of HIS mice can be generated with human FL-derived CD34 ${ }^{+} \mathrm{HSC}$ : HIS mice with no thymus, with a native mouse thymus, with a human thymus graft, or with both human and mouse thymi. To generate HIS mice with no thymus, NSG mice are thymectomized and 2 weeks later, sublethaly irradiated (100cGy) and injected i.v. with 1-2x $10^{5}$ human FL-derived CD34 ${ }^{+} \mathrm{HSC}$. HIS mice with a native mouse thymus are generated similarly, except they were not thymectomized. Similar to the first group, HIS mice with a human thymus graft are thymectomized. Two weeks later, the mice are injected i.v. with HSCs and transplanted with autologous (to human HSCs) human fetal thymus fragments measuring about $1 \mathrm{~mm}^{3}$ under the kidney capsule. HIS mice with both human and mouse thymi are generated similar to the HIS mice with a human thymus, except they were not thymectomized.

6 To ensure that pre-existing T cells in the transplanted donor human thymus are not able to persist, we freeze and thaw the thymus tissues and also pipett them up and down several times before transplantation to release as many thymocytes as possible. To further deplete passenger thymocytes that might migrate to the periphery, an anti-human CD2 antibody is injected to the mice in 2 weekly doses $(400 \mu \mathrm{g} / \mathrm{mouse}$, intraperitoneally).

7 HIS mice humanized at the neonatal stage are generated by injecting FL HSCs into neonates 1-2 days after birth (intra-liver, $10^{5} \mathrm{HSC}$ in $50 \mu \mathrm{PBS}$ injected using a 28-gauge needle). Neonatal mice receive half of the irradiation dose (50cGy) that the adult mice receive (100cGy). 
8 To generate Personalized Immune (PI) HIS mice with a human thymus graft, thymectomized NSG mice aresublethally irradiated (100cGy) and injected i.v. with $2 \times 10^{5}$ bone marrow (BM)derived $\mathrm{CD} 34^{+} \mathrm{HSC}$ from an adult donor. Two weeks after thymectomy, the mice are transplanted with human fetal thymus fragments (matched to the BM HSCs for a class I and a class II HLA allele) measuring about $1 \mathrm{~mm}^{3}$ under the kidney capsule. Similar to generation of mice with fetal HSCs, measures are applied to deplete the pre-existing thymus graft-derived T cells. 